\title{
Satélite Sino-Brasileiro de Recursos Terrestres na literatura científica global: tendências a partir de uma análise cienciométrica
}

\section{China-Brazil Earth Resources Satellite on global scientific literature: trends using scientometric analysis}

\section{Satélite Chino-Brasileño de Recursos Terrestres en la literatura científica mundial: tendencias de un análisis cienciométrico}

(iD) Patrick Thomaz de Aquino Martins

Universidade Estadual de Goiás - Formosa - Goiás -Brasil patrick.tdam@gmail.com

(iD) Pedro Paulino Borges

Universidade Estadual de Goiás - Anápolis - Goiás - Brasil borgespep@gmail.com

Resumo: Fruto da cooperação entre o Brasil e a China, o CBERS (Satélite Sino-Brasileiro de Recursos Terrestres) é um programa ao desenvolvimento de satélites de sensoriamento remoto o qual tem contribuído ao entendimento de diversos fenômenos e ao gerenciamento territorial. Embora haja produção de conhecimento a partir de dados do programa CBERS, esta ainda não foi caracterizada na literatura científica global. Desse modo, o objetivo do trabalho foi avaliar, por meio de uma análise cienciométrica, as tendências e lacunas dos estudos relacionados ao uso do CBERS. Considerando a base completa da plataforma ISI Web of Knowledge, foram pesquisados manuscritos que utilizaram ou analisaram o CBERS, resultando em 216 artigos, os quais foram examinados com base em dez diferentes critérios. Foi observado um aumento significativo de artigos ao longo do tempo. O satélite CBERS-2 foi o mais 
utilizado, com o CCD sendo o mais frequente dentre os todos os sensores. Quando se empregou outro produto de sensoriamento remoto, os satélites do programa Landsat e os das plataformas Aqua e Terra (sensor MODIS) foram os mais observados. Foi identificado um viés relacionado à nacionalidade dos países mantenedores do programa, assim como a prevalência do idioma inglês. O ambiente terrestre foi onde se deu a maior parte dos estudos, ocasionando algumas lacunas a serem exploradas. Além do entendimento de como a comunidade científica internacional vem utilizando o CBERS à geração de conhecimento, a análise cienciométrica identificou o cumprimento do propósito pelo qual o programa foi concebido, a autonomia na geração de dados orbitais.

Palavras-chave: CBERS. Cienciometria. Sensoriamento Remoto.

Abstract: As a result of the cooperation between Brazil and China, the China-Brazil Earth Resources Satellite (CBERS) is a program for the development of remote sensing satellites which has contributed to the understanding of various phenomena and territorial management. Although there is knowledge production from CBERS data, it has not been characterised within the global scientific literature. Thus, the objective of this study was to evaluate, through a scientometric analysis, the trends and gaps in studies related to the use of CBERS. Considering the ISI Web of Knowledge platform, manuscripts that used or analysed CBERS were searched, resulting in 216 articles, which were examined based on ten different criteria. A significant increase in articles has been observed over time. The CBERS-2 satellite was the most widely used, with CCD being the most frequent of all sensors. When using another remote sensing product, Landsat and Aqua/Terra satellites (MODIS sensor) were the most observed. A bias related to the nationality of the program maintainers was identified, as well as the prevalence of the English language. The majority of studies took place in terrestrial environments, causing some gaps to be explored. In addition to understanding how the international scientific community has been using CBERS for knowledge generation, the scientometric analysis identified the fulfilment of the purpose for which the program was designed and the autonomy in the generation of orbital data.

Keywords: CBERS. Scientometric. Remote Sensing. 
Resumen: Resultado de la cooperación entre Brasil y China, CBERS (Satélite Chino-Brasileño de Recursos Terrestres) es un programa para el desarrollo de satélites de teledetección que ha contribuido a la comprensión de diversos fenómenos y la gestión territorial. Aunque el conocimiento se produce a partir de datos del programa CBERS, todavía esta no se ha caracterizado en la literatura científica global. Por lo tanto, el objetivo de este estudio fue evaluar, mediante un análisis sciométrico, las tendencias y las brechas en los estudios relacionados con el uso de CBERS. Teniendo en cuenta la plataforma ISI Web of Knowledge, se realizaron búsquedas en manuscritos que utilizaron o analizaron CBERS, lo que resultó en 216 artículos, que fueron examinados en base a 10 criterios diferentes. Se ha observado un aumento significativo en los artículos con el tiempo. El satélite CBERS-2 fue el más utilizado, siendo CCD el más frecuente entre todos los sensores. Al usar otro producto de teledetección, los satélites Landsat y Aqua/Terra (sensor MODIS) fueron los más observados. Se identificó un sesgo relacionado con la nacionalidad de los encargados del mantenimiento del programa, así como la prevalencia del idioma inglés. El ambiente terrestre fue donde se realizaron la mayoría de los estudios, lo que provocó la exploración de algunas lagunas. Además de comprender cómo la comunidad científica internacional ha estado utilizando CBERS para la generación de conocimiento, el análisis cienciométrico identificó el cumplimiento del propósito para el que se diseñó el programa, la autonomía en la generación de datos orbitales.

Palabras clave: CBERS. Cienciometría. Teledetección.

Recebido para a publicação em 27 de abril de 2020

Aceito para a publicação em 10 de maio de 2020

Publicado em 
Satélite Sino-Brasileiro de Recursos Terrestres na literatura científica global...

Patrick Thomaz de Aquino Martins . Pedro Paulino Borges

\section{Introdução}

O CBERS (China-Brazil Earth Resources Satellite) é um programa de cooperação estabelecido entre a República Federativa do Brasil e a República Popular da China, iniciada com a assinatura de um protocolo, em 1988, para o desenvolvimento de uma família de satélites de sensoriamento remoto a ser executado, conjuntamente, pelas respectivas agências espaciais, a Nacional Institute for Space Research (INPE) e a Chinese Academy of Space Technology (CAST) (LINO et al., 2000).

Permitindo, inicialmente, o desenvolvimento e a construção de dois satélites de sensoriamento remoto, os quais foram denominados CBERS-1 e CBERS-2, a parceria resultou, até o momento, na construção de seis satélites, sendo necessária, contudo, a firmação de dois novos acordos, um para a construção de dois satélites com sensores mais avançados, CBERS-3 e CBERS-4, e outro para o lançamento de um satélite, batizado de CBERS-2B, que preencheria $O$ lapso temporal que haveria entre a vida útil dos dois primeiros e $O$ lançamento dos dois últimos satélites (NOVO, 2010).

Enquanto os três primeiros satélites CBERS conseguiram cumprir o tempo de vida projetado, o quarto satélite (CBERS-3) não foi colocado em órbita prevista, resultado de uma falha em seu veículo lançador. Atualmente, dois satélites estão em funcionamento, 0 CBERS-4, o qual teve seu lançamento antecipado devido à referida falha ocorrida com o CBERS-3, e o CBERS-4A, fruto de um novo protocolo assinado entre os países responsáveis pelo programa e que tem a missão de evitar ou minimizar um novo hiato no fornecimento de imagens CBERS (INPE, 2020).

Com características semelhantes aos satélites dos programas LANDSAT e SPOT (FLORENZANO, 2002; ROCHA, 2007; POWELL et al., 2007), o CBERS tem sido utilizado para diversas aplicações ligadas aos recursos terrestres, tais como para a classificação e monitoramento das condições de crescimento de culturas, avaliação de recursos terrestres, monitoramento de danos e riscos geológicos e à investigação e monitoramento de ecossistemas (YULIANG et al., 
Satélite Sino-Brasileiro de Recursos Terrestres na literatura científica global... Patrick Thomaz de Aquino Martins . Pedro Paulino Borges

2009). Além destas, as quais fazem jus à denominação do próprio CBERS, alguns estudos têm focado no entendimento e nas potencialidades do emprego das técnicas de sensoriamento remoto (e.g. MARTINS et al., 2009, BENSEBAA et al., 2014; PINTO et al., 2016) ou em temáticas não relacionadas especificamente aos recursos terrestres (e.g. CLARK, 2002, GARCIA et al., 2012; HONG et al., 2006).

Esta diversidade de aplicação, porém, ainda não foi analisada quanto aos aspectos quantitativos, disseminação e uso da informação registrada, ênfases que, de acordo com Strehl e Santos (2002), estão no âmbito da bibliometria e da cienciometria. A cienciometria e a bibliometria formam, juntamente com a webmetria, o conjunto de campos denominado informetria e possibilitam, dentre outras aplicações: identificar as tendências e o crescimento do conhecimento em uma área; identificar as revistas do núcleo de uma disciplina; prever as tendências de publicação; estudar a dispersão e a obsolescência da literatura científica; prever a produtividade de autores individuais, organizações e países; analisar os processos de citação e co-citação; avaliar os aspectos estatísticos da linguagem, das palavras e das frases; medir o crescimento de determinadas áreas e o surgimento de novos temas e detectar as lacunas de conhecimento sobre um determinado assunto (VANTI, 2002).

De natureza interdisciplinar, a pesquisa cienciométrica possui um escopo metodológico que utiliza métodos das ciências sociais, do comportamento e das ciências naturais. Além disso, faz uso de modelos de rede sociológica, levantamento psicológico, entrevistas, ciência da comutação e campos correlatos, além da estatística e outros métodos matemáticos (VAN RAAN, 1997). Até o presente momento não há um trabalho publicado que caracterize a literatura científica com ênfase em sensoriamento remoto, especificamente voltado para os artigos publicados utilizando informações provenientes do CBERS. Deste modo, o presente manuscrito tem por objetivo realizar uma análise cienciométrica, na literatura científica global, destacando as principais tendências e lacunas dos es- 
Satélite Sino-Brasileiro de Recursos Terrestres na literatura científica global...

Patrick Thomaz de Aquino Martins . Pedro Paulino Borges

tudos relacionados ao uso do Satélite Sino-Brasileiro de Recursos Terrestres.

\section{Material e Métodos}

A partir da plataforma ISI Web of Knowledge (Thomson Reuters Web of Science) foram pesquisados, considerando a coleção completa, manuscritos que utilizaram ou analisaram o CBERS. Para tal, foram buscados artigos que contivessem no título, resumo e/ou palavras-chave (busca tópica), os seguintes indexadores: "ChinaBrazil Earth Resources Satellite" OR "CBERS*”. A busca foi feita para captar artigos publicados durante todo o período disponível na plataforma (1945 e 2018). A Web of Science foi utilizada por ser a base de dados de maior importância no contexto científico, devido à qualidade, quantidade e registro histórico dos periódicos indexados, além da sistemática organização das informações pertinentes à análise cienciométrica.

Para cada manuscrito, foram consideradas as seguintes informações: (a) ano de publicação; (b) periódico; (c) localidade geográfica do estudo, por país; (d) abrangência geográfica (local, regional ou continental); (e) país de vínculo do primeiro autor, atribuindo aos demais autores o caráter de coautores, quando houve; $(f)$ satélite CBERS (1, 2, 2B, 3 ou 4) presente no manuscrito; (g) sensor CBERS utilizado; (h) outro programa (ou plataforma) de sensoriamento remoto empregado; (i) ambiente estudado (aquático, atmosférico ou terrestre); e (j) número de citações.

Para avaliar se o número de artigos que utilizaram o Satélite Sino-Brasileiro de Recursos Terrestres tem aumentado ao longo do tempo, foi realizada uma correlação de Pearson $(p<0,05)$ entre os anos e o total de artigos publicados e entre os anos e o número de artigos publicados em língua inglesa. As correlações foram feitas utilizando o programa R (R CORE TEAM, 2013). 
Satélite Sino-Brasileiro de Recursos Terrestres na literatura científica global... Patrick Thomaz de Aquino Martins . Pedro Paulino Borges

\section{Resultados}

A busca retornou 231 manuscritos indexados na plataforma ISI Web of Knowledge. Destes, 216 foram analisados e 15 foram descartados por razões diversas (apenas citar as palavras da busca, sem, de fato, utilizar o CBERS como parte do conteúdo; não tratar do satélite sino-brasileiro, e sim de outro tema com a mesma sigla; fazer parte de uma categoria não considerada, como a editorial).

O primeiro registro pertence ao ano de 1995, quatro anos antes do lançamento do primeiro satélite, enquanto o mais atual é de 2018, limite mais recente analisado. Em média, foram publicados 10,3 artigos por ano, sendo 2011 o ano com o maior número de publicação, 26, e 1997, 1998 e 1999 anos com ausência de artigos publicados (Figura 1). Foi observado, através da correlação, um aumento significativo de artigos ao longo do tempo $(r=0,67 ; p<$ 0,001), o mesmo padrão de aumento de artigos também foi observado para trabalhos publicados em Inglês $(r=0,72 ; p<0,001)$. 0 idioma mais utilizado nas publicações foi o inglês, com 169 (78,2\%) artigos, seguido por português, com $32(14,8 \%)$ artigos, chinês, com $9(4,2 \%)$ artigos, espanhol, com 5 (2,3\%), e polonês, com 1 $(0,5 \%)$ artigo. 
Satélite Sino-Brasileiro de Recursos Terrestres na literatura científica global... Patrick Thomaz de Aquino Martins - Pedro Paulino Borges

Figura 1 - Variação temporal do número de artigos publicados e artigos em Inglês que utilizaram o CBERS e estão indexados no ISI Web of Knowledge entre 1995 e 2018.

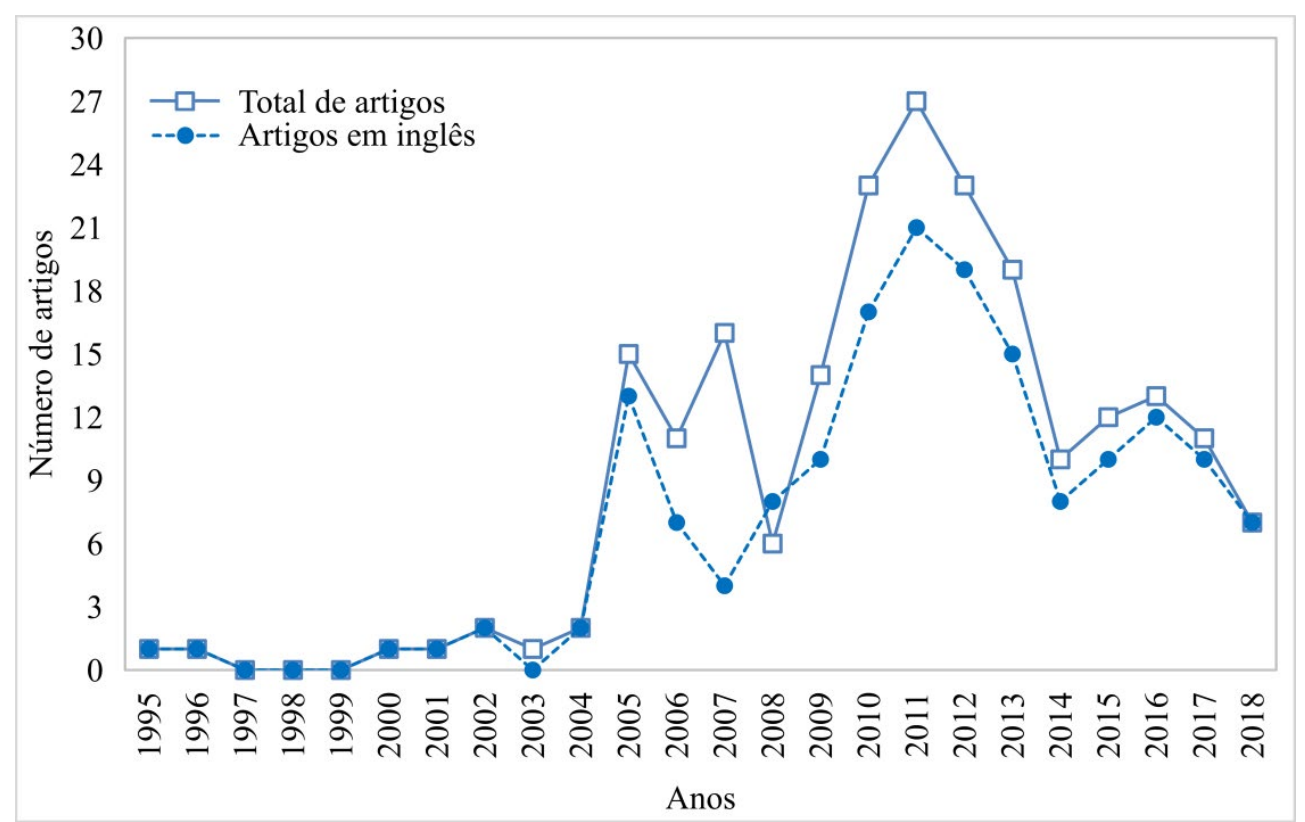

Foram identificados artigos em 102 diferentes periódicos, dos quais cerca de um quarto, $24,5 \%$, está contido em seis títulos, sendo o International Journal of Remote Sensing o periódico com o maior número de artigos, 15, seguido do Science in China Series E-Technological Sciences, com 11 artigos, e do Boletim de Ciências Geodesicas, com nove artigos (Figura 2). 
Satélite Sino-Brasileiro de Recursos Terrestres na literatura científica global... Patrick Thomaz de Aquino Martins . Pedro Paulino Borges

\section{Figura 2 - Periódicos com o maior número de artigos que utilizaram o CBERS e} estão indexados no ISI Web of Knowledge, entre 1995 e 2018.

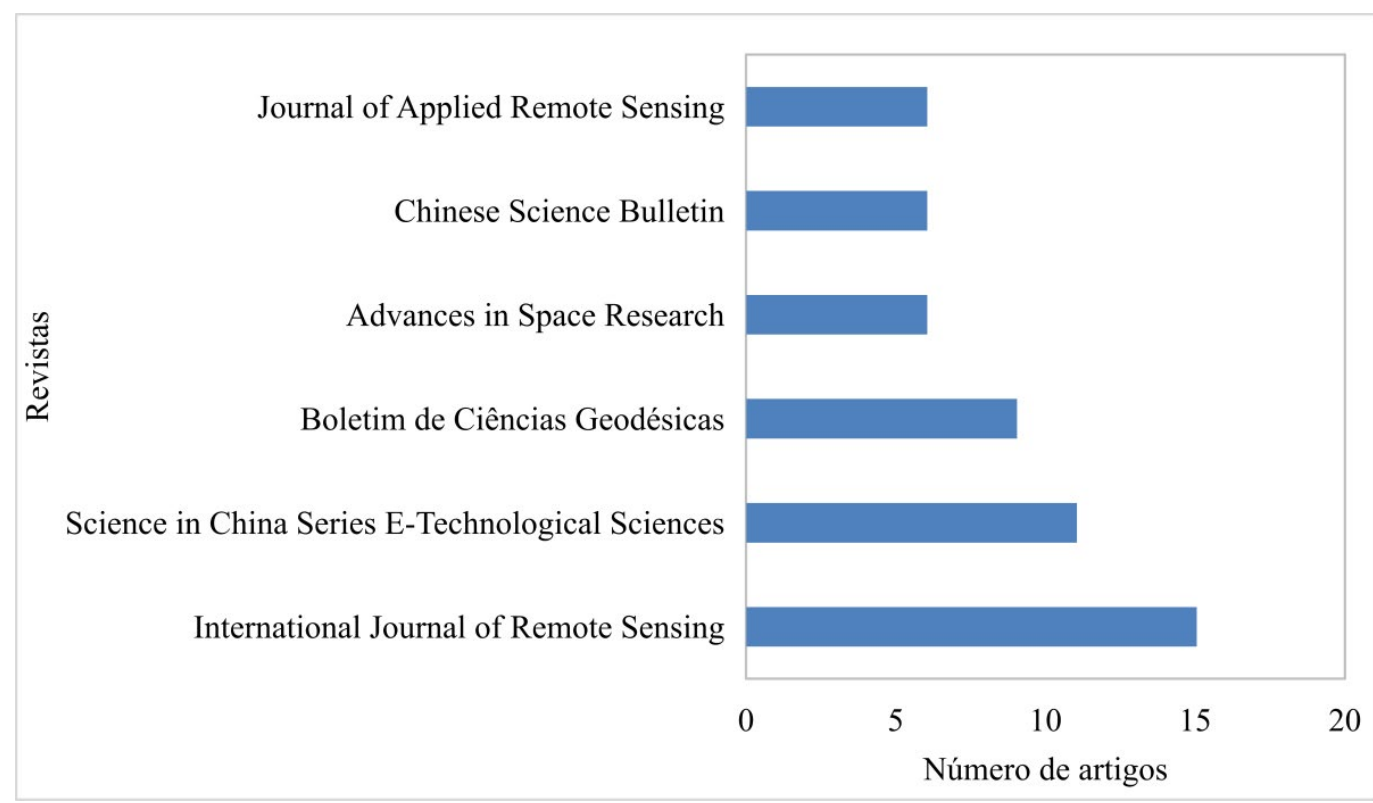

Embora tenham ocorridos autores vinculados a outros 14 países (Alemanha, Argentina, Bolívia, Bulgária, Canadá, Espanha, EUA, França, Holanda, Índia, Itália, Reino Unido, Rússia e Ucrânia), a maior parte dos artigos (82,3\%) foi de autoria de pesquisadores filiados a instituições na China (44,3\%) ou no Brasil (38\%) (Figura 3).

Em se tratando das localidades estudadas, é notada configuração similar, com $43,8 \%$ dos artigos tendo a China como espaço ao uso do CBERS (considerando um único país) e 37,3\% o Brasil. Ainda aparecem, com menor participação, artigos que tiveram como área de estudos porções das seguintes nações: Argentina, Bolívia, Canadá, Colômbia, Espanha, EUA, Tailândia e Venezuela. Há, do mesmo modo, trabalhos realizados em mais de uma nação e/ou em localidades de domínio público internacional, como a Antártida, o espaço ou as águas internacionais. Vale ressaltar que a participação aqui apontada somente considera artigos que fizeram uso de algum território à aplicação de imagens do sensor, ignorando, por exemplo, artigos relacionados à calibração pré-lan- 
Satélite Sino-Brasileiro de Recursos Terrestres na literatura científica global... Patrick Thomaz de Aquino Martins . Pedro Paulino Borges

çamento, à atitude do satélite, ao desenvolvimento de tecnologia de soldagem ou àqueles identificados como de revisão.

Figura 3 - Distribuição Geográfica da produção científica que utilizou o CBERS e estão indexados no ISI Web of Knowledge, entre 1995 e 2018.

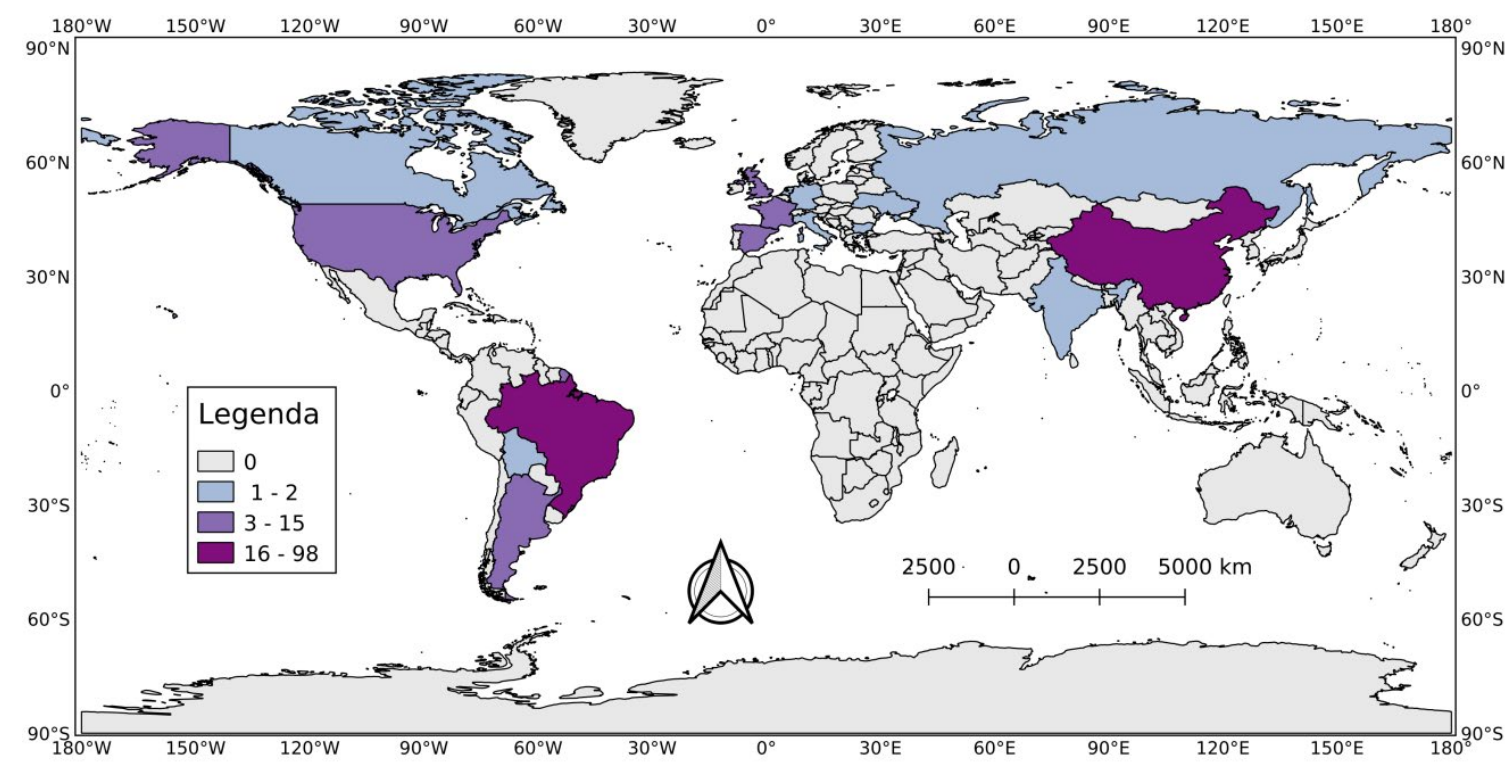

Utilizando-se do mesmo critério, foi possível observar que a maior parte dos estudos foi realizada em uma reduzida porção geográfica, havendo predomínio da escala local em 85,9\% dos trabalhos. Apenas $8,1 \%$ dos artigos compreenderam a extensão regional e 5,9\% a escala continental, não se aplicando a este tipo de análise 16,76\% dos 216 manuscritos analisados.

Na maioria dos periódicos, 137, foi informada a utilização de outros programas de sensoriamento remoto, tais como Spot, Ikonos, Alos, Geoeye e RapidEye, havendo, também, ocorrência de uso de VANT, fotografia e videografia aéreas, além de equipamento não imageador, como o espectrorradiômetro. Os programas Landsat e Terra-Aqua (sensor MODIS - Moderate-Resolution Imaging Spectroradiometer) foram os programas com maior quantidade de ocorrência contígua ao CBERS, presentes, respectivamente, em 81 
Satélite Sino-Brasileiro de Recursos Terrestres na literatura científica global... Patrick Thomaz de Aquino Martins . Pedro Paulino Borges

e 35 artigos, i.e., 59,1\% e 25,5\% dentre os manuscritos que fizeram uso de outro programa além do CBERS, ou $37,5 \%$ e $16,2 \%$ do total dos trabalhos.

Dentre os satélites do programa CBERS, o mais utilizado foi o CBERS 2 (49,1\%), seguido pelo CBERS 2B (29,5\%), pelo CBERS 1 $(16,9 \%)$ e pelo CBERS 4 (3,9\%). Com menor participação aparece o CBERS 3, com somente um, 0,4\%, dos artigos (Figura 4A). Em relação aos sensores, quando este é observado, o CCD é, disparado, o mais utilizado, presente em 137 artigos (74,1\%). Os demais sensores participam com os seguintes percentuais: HRC com 13\%, WFI com 4,3\%, IRMSS com 3,8\%, MUX e PAN com 2,2\% e IRS com 0,5 (Figura 4B).

Figura 4 - Participação dos satélites (A) e sensores (B) CBERS nos artigos que utilizaram o CBERS e estão indexados no ISI Web of Knowledge, entre 1995 e 2018.

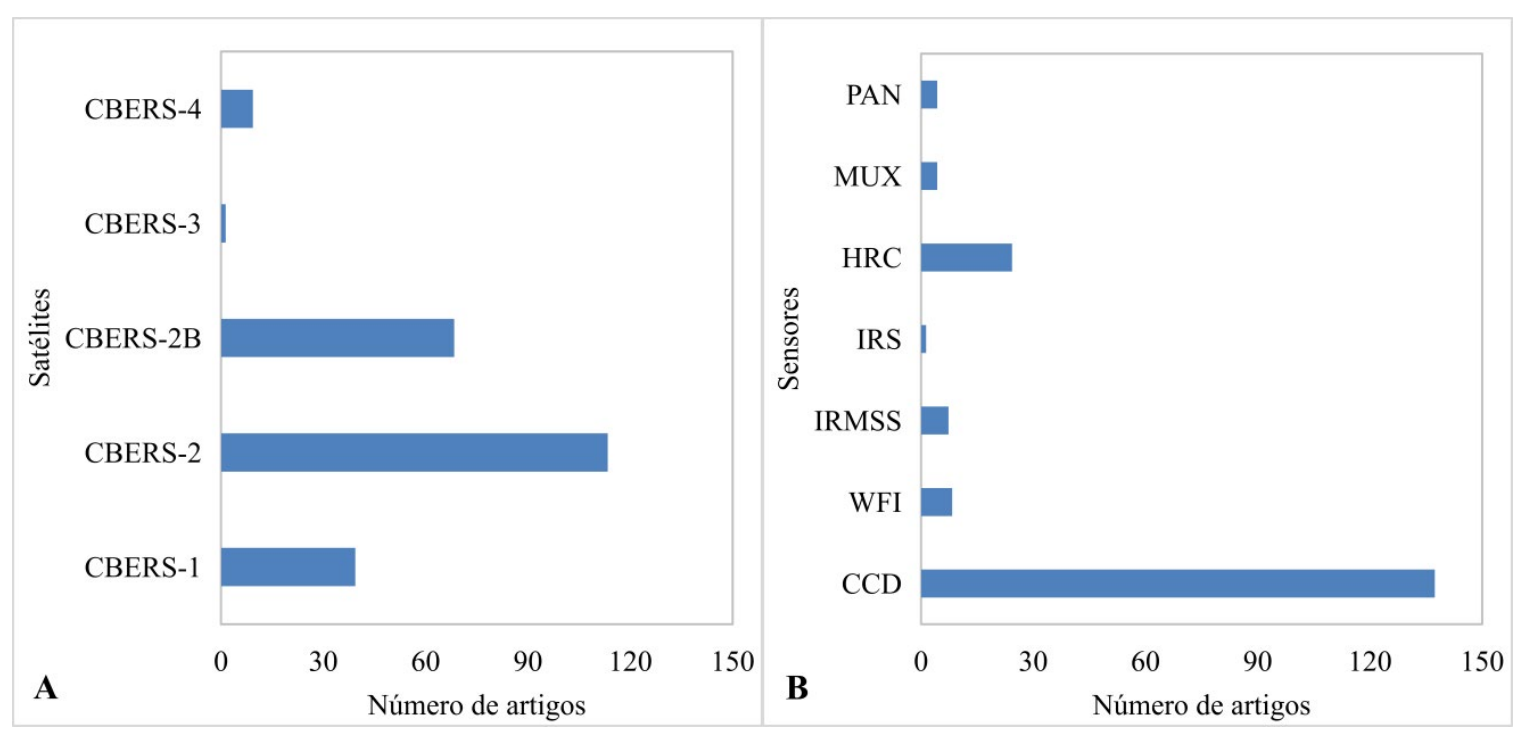

A maior parte dos trabalhos, 101 (70,1\%), foi desenvolvida no contexto do ambiente terrestre. Neste caso, foram contabilizados tanto artigos cujo objetivo focavam analisar o ambiente em si (e.g. mapeamento de cobertura florestal, estimativa de lavoura e sus- 
Satélite Sino-Brasileiro de Recursos Terrestres na literatura científica global...

Patrick Thomaz de Aquino Martins . Pedro Paulino Borges

cetibilidade a movimento de massa), quanto trabalhos cujos cerne foi o desenvolvimento, validação ou avaliação de alguma técnica intrínseca ao sensoriamento remoto, mas se utilizaram ou foram voltadas ao ambiente terrestre. Com quantitativo bem abaixo aparecem os trabalhos realizados em ambientes aquáticos e atmosféricos, com 36 (25\%) e sete $(4,9 \%)$, respectivamente.

No que tange a quantidade de vezes que o trabalho é citado, foi constatado que $29(13,4 \%)$ artigos ainda não possuem citação, sendo que 14 destes foram publicados há três anos ou menos, ao passo que $152(70,4 \%)$ manuscritos possuem menos de dez citações. No extremo oposto, os dez artigos mais citados totalizam 1.039 citações, com destaque ao trabalho de Crétaux et al. (2011), único com mais de 200 citações (Tabela 1).

\section{Tabela 1 - Artigos com participação do CBERS mais citados na base ISI Web of Knowledge, entre 1995 e 2018.}

\begin{tabular}{lllll}
\hline Autores & Título & Periódico & Ano & Citações \\
\hline \multirow{2}{*}{$\begin{array}{l}\text { Cretaux et } \\
\text { al. }\end{array}$} & SOLS: A lake database to monitor in the & & & \\
& Near Real Time water level and storage & ADVANCES IN SPACE & 2011 & 214 \\
& variations from remote sensing data & RESEARCH & &
\end{tabular}

Ma et al China's lakes at present: Number, area and SCIENCE CHINA-EARTH spatial distribution

SCIENCES

$2011 \quad 127$

IEEE JOURNAL OF SE-

A Global Human Settlement Layer From LECTED TOPICS IN

Pesaresi Optical HR/VHR RS Data: Concept and First APPLIED EARTH OBSER-

$2013 \quad 119$

Results

VATIONS AND REMOTE

SENSING

Shen e A MAP-Based Algorithm for Destriping and

IEEE TRANSACTIONS

Zhang Inpainting of Remotely Sensed Images

ON GEOSCIENCE AND

2009

118

REMOTE SENSING

Assessing the effects of urbanization on

Du et al. annual runoff and flood events using an integrated hydrological modeling system

JOURNAL OF HYDROL-

OGY

2012

113

for Qinhuai River basin, China

Niu et al. Mapping wetland changes in China between 1978 and 2008

CHINESE SCIENCE BULLETIN 
Satélite Sino-Brasileiro de Recursos Terrestres na literatura científica global... Patrick Thomaz de Aquino Martins . Pedro Paulino Borges

Evolving neural network using real coded

Liu et al. genetic algorithm (GA) for multispectral image classification

FUTURE GENERATION COMPUTER SYSTEMS

Leaf area index estimation with MODIS

le Maire reflectance time series and model inver-

et al.

sion during full rotations of Eucalyptus plantations

REMOTE SENSING OF ENVIRONMENT

Chuvieco Global burned-land estimation in Latin et al. America using modis composite data

ECOLOGICAL APPLICATIONS

RomeLandscape transformations in savannas of ro-Ruiz et northern South America: Land use/cover al. changes since 1987 in the Llanos Orientales of Colombia

\section{Discussão}

O objetivo do presente trabalho foi avaliar as principais tendências e lacunas dos estudos relacionados ao uso do Satélite Sino-Brasileiro de Recursos Terrestres (CERBS). Foi observado que o número de artigos sobre o tema tem aumentado significativamente ao longo do tempo. O aumento do número de artigos com o CBERS corrobora a tendência global no uso de sensoriamento remoto em estudos científicos, conforme identificado por Vijaylakshmi e Ambuja (2013), Zhuang et al. (2013) e Hanqing et al. (2016). Esta, seguramente, acompanha o crescimento da produção na literatura científica como um todo, pois trabalhos em diversas áreas (e.g. BORGES et al., 2015; COSTA et al., 2018) têm registrado aumento no número de publicações ao longo dos anos.

Há pouco mais de uma década Powell et al. (2007) indicavam que aplicações documentadas de imagens CBERS eram difíceis de serem encontradas na literatura primária. Ainda que este quadro tenha melhorado, como pode ser notado no presente trabalho, alguns procedimentos fundamentais a utilizações mais avançadas das imagens, como a correção atmosférica, ainda não foram amplamente parametrizados/modelados para as imagens CBERS. A presença em alguns periódicos de referência na área de sensoria- 
Satélite Sino-Brasileiro de Recursos Terrestres na literatura científica global...

Patrick Thomaz de Aquino Martins . Pedro Paulino Borges

mento remoto, como o International Journal of Remote Sensing, o qual registrou o maior número de artigos que utilizaram o CBERS, pode auxiliar na divulgação dos usos e potencialidades das imagens CBERS, oportunizando, à comunidade científica internacional, possibilidade de desenvolvimento de técnicas e aplicações ainda não testadas nas imagens CBERS.

A despeito de ser uma revista tradicional na área, a quantidade de números publicados anualmente pela International Journal of Remote Sensing, 24, a partir de 2002, parece influenciar na posição alcançada por este periódico quanto à presença de trabalhos com CBERS. Vale ressaltar, ainda, que, dentre as seis revistas com o maior número de artigos, metade é constituída de periódicos editados ou pertencentes a alguma organização da China ou do Brasil, o que demonstra um viés que pode estar associado aos países mantenedores do programa de satélite.

A predominância do idioma inglês como o mais utilizado nos artigos, assim como o aumento deste ao longo do tempo analisado, se justifica pelo fato de, além de ser língua franca da ciência (MENEGHINI; PACKER, 2007), neste idioma, o trabalho se tornar acessível a um público maior, resultando em maior número de citação (BITETTI; FERRERAS, 2017). A utilização do português e do chinês está nitidamente associado ao idioma nativo dos autores, concomitante à nacionalidade do periódico indexado na base avaliada.

Presença frequente nos dois primeiros lugares em diversos levantamentos ligados à produção de papers (e.g. THOMSON REUTERS, 2012; SCIMAGO, 2007), inclusive em pesquisas vinculado ao sensoriamento remoto (ZHUANG et al., 2013; SARRAGIOTTO; BENEDITO, 2013), a participação da China como maior contribuidora de trabalhos científicos que abordem o CBERS não é surpresa. A posição do Brasil, porém, ratifica a tendência existente entre os pesquisadores naturais ou radicados em um dos países desenvolvedores do CBERS em utilizarem informações sobre o referido programa em trabalhos científicos. Uma vez que a coleta de dados de referência terrestre in situ é parte intrínseca do processo do 
Satélite Sino-Brasileiro de Recursos Terrestres na literatura científica global... Patrick Thomaz de Aquino Martins . Pedro Paulino Borges

Sensoriamento Remoto (JENSEN, 2009), e que há predominância de autores oriundos da China e do Brasil, é razoável que a maior parte dos estudos ocorra nos territórios destes países, sobretudo considerando a redução dos custos à obtenção dos dados.

A mesma linha de entendimento pode ser atribuída à elevada quantidade de artigos de abrangência local. Nesta escala de análise, aqui identificado como trabalhos cujos escala geográfica abarcou áreas iguais ou menores que um estado ou província, ao que pese a extensão territorial dos países com maior produção de estudos com CBERS, os custos à realização das etapas in situ são bastante reduzidos, quando comparado a estudos em escalas regionais (estados ou países) ou continentais (países e planeta), principalmente em trabalhos experimentais.

As tentativas de se entender as potencialidades das imagens CBERS às diferentes aplicações de dados de sensoriamento remoto explica, em parte, o uso de dados provenientes de outros programas de sensoriamento remoto. Esta abordagem é comum em análises experimentais, onde se busca testar a aplicabilidade das imagens a determinados estudos, ou de técnicas à esta, utilizando-se de outras imagens como referência (e.g. ZHANG, TANG; DONG, 2010; DU et al., 2012; TAO et al., 2013; BOGGIONE et al., 2014). Como foram analisados artigos contidos em uma base que se destaca pela sua associação à relevância internacional, é de se esperar que a maior parte dos trabalhos se fundamente em programas consolidados.

A justificativa que complementa a utilização do CBERS concomitante a outros sensores/programas é a necessidade de se complementar alguma característica ausente em uma das imagens, como as associadas às resoluções (espacial, espectral ou temporal). A predileção pelo programa Landsat é explicada não apenas pela semelhança, já citada, entre o CBERS e o Landsat, mas também por este estar entre os satélites mais amplamente utilizado como fonte de dados em diversos tipos de estudos, tais como aqueles relacionados a recursos hídricos (FEYISA et al., 2014), à cobertura da terra (KNORN et al., 2009) e a temperatura de superfície (LIU; 
Satélite Sino-Brasileiro de Recursos Terrestres na literatura científica global...

Patrick Thomaz de Aquino Martins . Pedro Paulino Borges

ZHANG, 2011), o que se justifica pelas quase cinco décadas nas quais as imagens Landsat têm sido postas à prova em diferentes áreas do conhecimento.

Não tão longevo quanto o Landsat, fornecendo dados desde o ano 2000, os satélites os quais possuem o sensor MODIS a bordo, denominados Terra e Aqua, se notabilizam por prover dados com tempo de revisita entre um e dois dias, além de serem rigorosamente validado e amplamente utilizado em diversas aplicações (GAO et al., 2015). Os dados satelitários com alta resolução temporal, como MODIS, sempre estão associados a imagens com baixa resolução espacial (WU et al., 2016), o que explica a utilização de imagens CBERS com aquelas provenientes do MODIS.

A relativa equivalência do sensor $C C D$, presente nos três primeiros satélites do programa sino-brasileiro, com os "Thematic Mapper" (TM e Enhanced Thematic Mapper Plus - ETM+), da série de satélites Landsat, esclarece a presença deste sensor como o CBERS mais utilizado. Soma-se a esta similaridade o fato de a resolução espacial destes sensores serem destinadas a fornecer escala adequada para uma ampla variedade de aplicações de recursos terrestres (ROGAN; CHEN, 2004).

À semelhança do Landsat, as imagens CCD têm sido utilizadas ao mais diversos fins, tais como à análise do uso e cobertura da terra (ZHENG et al., 2012; WANG et al., 2012), avaliação de eventos de inundação (MENDONÇA et al., 2012), estimativa de área cultivada (LI et al., 2011) e concentração de matéria suspensa (ZHANG et al., 2010). A ampla predileção pelas imagens do sensor CCD, associada ao tempo de vida útil, refletiu, e justifica, a posição do segundo satélite da série, o CBERS-2, como o mais recorrente dentre os trabalhos analisados.

A propensão pela aplicação do CBERS ao ambiente terrestre pode estar relacionada à diversidade das áreas de aplicação que se utilizam do sensoriamento remoto para produção de conhecimento neste meio, tais como agricultura, geologia, florestal e de estudos urbanos, além de temas transversais eminentemente 
Satélite Sino-Brasileiro de Recursos Terrestres na literatura científica global...

Patrick Thomaz de Aquino Martins . Pedro Paulino Borges

ligados a este ambiente, a exemplo do fogo e do uso da terra, pluralidade que não é possível aos ambientes aquáticos e atmosférico. Mesmo não sendo o ambiente de maior aplicação do CBERS, o meio aquático se destaca como principal ambiente dentre os artigos que recebem maior número de citações (top 10), com quatro dos seis artigos com mais de 100 citações, e o único com mais de 200 citações.

No tocante às citações, as informações contidas na Tabela 1 refletem alguns padrões já conhecidos em bibliometria. Artigos com mais de quatro autores, por exemplo, tendem a receber mais citações que a média (HSU; HUANG, 2011), como é o caso de nove dos dez artigos com mais de 100 citações. Em todos os artigos o idioma utilizado foi o inglês, o que, como já informado, também está associado a um maior número de citações (BITETTI; FERRERAS, 2017). Mesmo sendo o segundo país com maior presença de autores e localidade de trabalhos, nenhum trabalho cujo autoria ou localidade é brasileira figura entre os 10 artigos mais citados (todos da Tabela 1), corroborando o que foi identificado por Meneghini et al. (2008), os quais apontaram que artigos com autores brasileiros, bem como de outros nacionalidades da América Latina, em revistas prestigiadas recebem menos citações. A efeito de exemplo, no presente trabalho, o primeiro artigo de autoria brasileira figura somente na $30^{a}$ posição. Para além das informações contidas além da Tabela 1, foi possível observar, ainda, a baixa ou ausência de citação da maioria dos artigos, paradigma também já identificado na comunidade científica (COLQUHOUN, 2003).

\section{Considerações finais}

Estar vinculado a um dos países mantenedores do programa CBERS parece influenciar na utilização dos satélites e imagens em estudos publicados em revistas de qualidade reconhecida pela Web of Science, uma vez que China e Brasil monopolizaram as variáveis relacionadas à nacionalidade. A manutenção do crescimento no 
Satélite Sino-Brasileiro de Recursos Terrestres na literatura científica global... Patrick Thomaz de Aquino Martins . Pedro Paulino Borges

uso do inglês como idioma de publicação tende a tornar o CBERS mais conhecido a partir da literatura científica internacional, oportunizando o desenvolvimento de técnicas e a exploração de aplicações, ainda não contempladas ou não consolidadas, às imagens e satélites do programa.

No que concerne aos recursos terrestres, por exemplo, a pouca atenção dada aos ambientes aquáticos e atmosféricos pode ser considerada uma lacuna, que deve ser explorada, na aplicação do CBERS a estes ambientes, sobretudo com a atenção, em termos de citação, que os trabalhos realizados no meio aquático demonstraram e da relevância contemporânea, na academia, de temas relacionados às mudanças climáticas.

A cienciometria permitiu entender como o CBERS tem sido utilizado à produção de conhecimento científico em veículos de alto prestígio acadêmico. O cenário aqui identificado tende a se manter o mesmo nos próximos anos, uma vez que, embora as imagens CBERS possam ser consideradas equivalentes, em termos qualidade, características e custo ao usuário, aos programas e missões de sensoriamento remoto amplamente utilizados na comunidade científica internacional, como o Landsat e o Sentinel, Ihes falta um diferencial (e.g. disponibilizar imagem em valores de reflectância de superfície ou um produto resultado de índice espectral) à real compatibilidade com seus pares. Se por um lado essa conjuntura possa denotar o pouco aproveitamento do CBERS pela comunidade científica internacional, por outro ratifica o investimento feito pelos países em busca da independência na geração de dados satelitários, emancipação significativa em diversos segmentos, tais como o tecnológico, o geopolítico e o ambiental.

\section{Agradecimentos}

PPB agradece à Coordenação de Aperfeiçoamento de Pessoal de Nível Superior (CAPES) - código de financiamento 001, pela 
Satélite Sino-Brasileiro de Recursos Terrestres na literatura científica global...

Patrick Thomaz de Aquino Martins . Pedro Paulino Borges

bolsa de doutorado concedida. Os autores agradecem a Matthijs Strietman pela revisão do idioma inglês.

\section{Referências}

BENSEBAA, K.; BANON, G. J. F.; FONSECA, L. M. G. Spatial resolutionestimation of CBERS-1 and CBERS-2 CCD cameras, International Journal of Remote Sensing, v. 33, n. 2, p. 604-629, 2012, doi: 10.1080/01431161.2010.545840.

BOGGIONE, G. A.; PEREIRA, G.; CARDOZO, F. S.; FONSECA, L. M. G. Evaluation of simulated images of MUX camera from CBERS-4 satellite for environmental analysis. Boletim de Ciências Geodésicas, v. 20, n. 3, p. 590-609, 2014.

BORGES, P. P.; OLIVEIRA, K. A. F. A.; MACHADO, K. B.; VAZ, Ú. L.; CUNHA, H. F.; NABOUT, J. C. Trends and gaps of the scientific literature on the Cerrado biome: A scientometric analysis.

Neotropical Biology and Conservation, v. 10, n. 1, p. 2-8, 2015.

CLARK, P. S. Orbital manoeuvres of China's Zi Yuan satellites. JBIS - Journal of the British Interplanetary Society, v. 55 p. 236-240, 2002.

COLQUHOUN, D. Challenging the tyranny of impact factors.

Nature, v. 423, n. 6939, p. 479, 2003.

COSTA, J. A.; SOUZA, J. P.; TEIXEIRA, A. P.; NABOUT, J. C.;

CARNEIRO, F. M. Eutrophication in aquatic ecosystems: a scientometric study. Acta Limnologica Brasiliensia, v. 30, e2, 2018.

CRÉTAUX, J. F.; JELINSKI, W.; CALMANT, S.; KOURAEV, A.; VUGLINSKI, V.; BERGÉ-NGUYEN, M.; GENNERO, M. C.; NINO, F.; ABARCA DEL RIO, R.; CAZENAVE, A.; MAISONGRANDE, P. Advances in Space Research, v. 47, n. 9, p. 1497-1507, 2011.

DI BITETTI, M. S.; FERRERAS, J. A. Publish (in English) or perish: The effect on citation rate of using languages other than English 
Satélite Sino-Brasileiro de Recursos Terrestres na literatura científica global...

Patrick Thomaz de Aquino Martins . Pedro Paulino Borges

in scientific publications. Ambio, v. 46, n. 1, p. 121-127, 2017, doi:10.1007/s13280-016-0820-7.

DU, P.; LIU, S.; GAMBA, P.; TAN, K.; XIA, J. Fusion of Difference Images for Change Detection over Urban Areas. IEEE Journal of Selected Topics in Applied Earth Observations and Remote Sensing, v. 5, n. 4, p. 1076-1086, 2012.

FEYISA, G. L.; MEILBY, H.; FENSHOLT, R.; PROUD, S. R. Automated Water Extraction Index: A new technique for surface water mapping using Landsat imagery. Remote Sensing of Environment, v. 140, p. 23-35, 2014.

FLORENZANO, T. G. Imagens de satélite para estudos ambientais. 1. ed. São Paulo: Oficina de Textos, 2002. 104p.

GAO, F.; HILKER, T.; ZHU, X.; ANDERSON, M.; MASEK, J.; WANG, P.; YANG, Y. Fusing Landsat and MODIS Data for Vegetation Monitoring. IEEE Geoscience and Remote Sensing Magazine, $v$. 3, n. 3, p. 47-60, 2015.

GARCIA, R. V.; KUGA, H. K.; ZANARDI, M. C. F. P. S. Unscented Kalman Filter Applied to the Spacecraft Attitude Estimation with Euler Angles. Mathematical Problems in Engineering, 2012, doi: 10.1155/2012/985429.

HANQING, M.; FENG, G.; XINYU, H.; HUI, L.; XIAOMEI, Y. Publication Trend of the Remote Sensing Technology and Application in Recent 30 Years based on Bibliometric Analysis. Remote Sensing Technology and Application, v. 31, n. 6, p. 1215-1222, 2016.

HONG, Z.; ZUO, X.; YONGQIANG, H.; JIQING, Z.; WENMING, Z.; ZHONGXIANG, W.; HONGWEN, X. Analysis of the observation of particle detector inside 'CBERS-1' satellite under solar quiet conditions. Science in China: Series E Technological Sciences, v. 49, n. 3, p. 342-357, 2006.

HSU, J.; HUANG, D. Correlation between impact and collaboration. Scientometrics, v. 86, n. 2, p. 317-324, 2011. 
Satélite Sino-Brasileiro de Recursos Terrestres na literatura científica global...

Patrick Thomaz de Aquino Martins . Pedro Paulino Borges

INPE. Instituto Nacional de Pesquisas Espaciais. CBERS. Disponível em: http://www.cbers.inpe.br/. Acesso em: 7 mai. 2020.

JENSEN, J. R. Sensoriamento Remoto do Ambiente: Uma

Perspectiva em Recursos Terrestres. São José dos Campos, SP: Parêntese, 2009. 598p.

KNORN, J.; RABE, A.; RADELOFF, V. C.; KUEMMERLE, T.; KOZAK, J.; HOSTERT, P. Land cover mapping of large areas using chain classification of neighboring Landsat satellite images. Remote Sensing of Environment, v. 133, n. 5, p. 957-964, 2009, doi: 10.1016/j. rse.2009.01.010.

LI, Q.; WU, B.; JIA, K.; DONG, Q.; EERENS, H.; ZHANG, M. Maize acreage estimation using ENVISAT MERIS and CBERS-02B CCD data in the North China Plain. Computers and Electronics in Agriculture, v. 78, n. 2, p. 208-214, 2011.

LINO, C. O.; LIMA, M. G. R.; HUBSCHER, G. L. CBERS - An international space cooperation program. Acta Astronautica, v. 47, n. 2-9, p. 559-564, 2000.

LIU, L.; ZHANG, Y. Urban Heat Island Analysis Using the Landsat TM Data and ASTER Data: A Case Study in Hong Kong. Remote Sensing, v. 3, n. 7, p. 1535-1552, 2011, doi: 10.3390/rs3071535.

MENDONÇA, J. C.; FREITAS, R. M.; SHIMABUKURO, Y. E.; MARQUES, V. S. Avaliação de eventos de inundação na Região Norte Fluminense, Rio de Janeiro, utilizando imagens de sensores remotos. Revista Ambiente e Água, v. 7, n. 1, p. 255-267, 2012.

MARTINS, P. T. A.; GRADELLA, F. S.; CORRADINI, F. A.; PUPIM, F. N.; COELHO, J. O. M. Análise comparativa entre a fusão de imagens CCD/HRC e TM/HRC na região dos Rios Miranda e Abobral, Pantanal Sul-mato-grossense. Geografia (Rio Claro. Impresso), v. 34, p. 807-815, 2009.

MENEGHINI, R; PACKER, A. L. Is there science beyond English? Initiatives to increase the quality and visibility of non-English publications might help to break down language barriers in scientific 
Satélite Sino-Brasileiro de Recursos Terrestres na literatura científica global...

communication. EMBO Reports, v. 8, n. 2, p. 112-116, 2007, doi: 10.1038/sj.embor.7400906.

MENEGHINI, R.; PACKER, A. L.; NASSI-CALÒ, L. Articles by latin american authors in prestigious journals have fewer citations. PLoS ONE, v. 3, n. 11, p. e3804, 2008.

NOVO, E. M. L. M. Sensoriamento remoto: princípios e aplicações. 4. ed. São Paulo, Blücher, 2010. 388p.

PINTO, C.; PONZONI, F.; CASTRO, R.; LEIGH, L.; MISHRA, N.; AARON, D.; HELDER, D. First in-Flight Radiometric Calibration of MUX and WFI on-Board CBERS-4. Remote Sensing, v. 8, p. 405, 2016.

POWELL, S. L.; PFLUGMACHER, D.; KIRSCHBAUM, A. A.; KIM, Y.; COHEN, W. B. Moderate resolution remote sensing alternatives: a review of Landsat-like sensors and their applications. Journal of Applied Remote Sensing, v. 1, n. 1, 16p., 2007.

R CORE TEAM. R: a language and environment for statistical computing. Vienna: R Foundation forStatistical Computing. Disponível em: http://www.R-project.org/. Acesso em: 5 out. 2013.

ROCHA, C. H. B. Geoprocessamento: tecnologia transdisciplinar. Juiz de Fora, MG: do autor, 3. ed., 2013. 220p.

ROGAN, J.; CHEN, D. Remote sensing technology for mapping and monitoring land-cover and land-use change. Progress in Planning, v. 61, n. 4, p. 301-325, 2004.

SARRAGIOTTO, M. C.; BENEDITO, E. Scientometric diagnosis of the use of remote sensing images in Landscape Ecology studies. Acta Scientiarum. Biological Sciences. Maringá, v. 35, n. 1, p. 41-46, 2013.

SCIMAGO. SCImago Journal \& Country Rank. SJR. Disponível em: http://www.scimagojr.com/countryrank.php. Acesso em: 16 mai. 2017. 
Satélite Sino-Brasileiro de Recursos Terrestres na literatura científica global...

Patrick Thomaz de Aquino Martins . Pedro Paulino Borges

STREHL, L.; SANTOS, C. A. Indicadores de qualidade da atividadecientífica. Ciência Hoje, Rio de Janeiro, v. 31, n. 186, p. 34-39, 2002.

THOMSON REUTERS. Global Publishing: Changes in submission trends and the impact on scholarly publishers. 2012. Disponível em: http://scholarone.com/media/pdf/GlobalPublishing_WP.pdf. Acesso em: 03 jul. 2017.

VAN RAAN, A. F. J. Scientometrics: state-of-the-art.

Scientometrics, v. 38, n. 1, p. 205-218, 1997.

VANTI, N. A. P. Da bibliometria à webometria: uma exploração conceitual dos mecanismos utilizados para medir o registro da informação e a difusão do conhecimento. Ciência da Informação, Brasília, v. 31, n. 2, p. 369-379, 2002.

VIJAYLAKSHMI, S.; AMBUJA, R. Remote Sensing literature in Scopus database: a bibliometric analysis. International Journal of Library and Information Studies, v. 3, n. 1, p. 70-86, 2013.

WANG, D.; GONG, J.; CHEN, L.; ZHANG, L.; SONG, Y.; YUE, Y. Spatio-temporal pattern analysis of land use/cover change trajectories in Xihe watershed. International Journal of Applied Earth Observation and Geoinformation, v. 14, n. 1, p. 12-21, 2012.

WU, M.; WU, C.; HUANG, W.; NIU, Z.; WANG, C.; LI, W.; HAO, P. An improved high spatial and temporal data fusion approach for combining Landsat and MODIS data to generate daily synthetic Landsat imagery. Information Fusion, v. 31, p.14-25, 2016.

YULIANG, Q.; SHANGMIN, Z.; ZHEN, L.; BEI, J. Application of ChinaBrazil Earth resources satellite in China. Advances in Space Research, v. 43, p. 917-922, 2009.

ZHANG, M.; TANG, J.; DONG, Q. Study on the cross-calibration of a charge-coupled device camera on the China-Brazil Earth Resources Satellite-02B by moderate resolution imaging spectroradiometer. Journal of Applied Remote Sensing, v. 4, n. 1, 043523, 2010. 
Satélite Sino-Brasileiro de Recursos Terrestres na literatura científica global...

Patrick Thomaz de Aquino Martins . Pedro Paulino Borges

ZHANG, M.; DONG, Q.; TANG, J.; SONG, Q. Evaluation of the retrieval of total suspended matter concentration in Taihu Lake, China from CBERS-02B CCD. Chinese Journal of Oceanology and Limnology, v. 28, n. 6, p. 1316-1322, 2010.

ZHENG, Z.; YANG, W.; ZHOU, G.; WANG, X. Analysis of land use and cover change in Sichuan province, China. Journal of Applied Remote Sensing, v. 6, n. 1, 2012.

ZHUANG, Y; LIU, X.; NGUYEN, T.; HE, Q.; HONG, S. Global remote sensing research trends during 1991-2010: a bibliometric analysis. Scientometrics, v. 96, p. 203-219, 2013.

\section{Declaração de contribuição individual}

Declaramos, para os devidos fins, que todos os autores ofereceram substanciais contribuições científicas e intelectuais ao estudo. As tarefas de concepção e design do estudo, preparação e redação do manuscrito, bem como, revisão crítica foram desenvolvidas em grupo. O primeiro autor, Patrick Thomaz de Aquino Martins, ficou especialmente responsável pelo desenvolvimento teórico-conceitual, aquisição dos dados e redação do texto; e o segundo autor, Pedro Paulino Borges, pela interpretação e análise dos dados e revisão crítica do texto.

Patrick Thomaz de Aquino Martins - Possui Graduação em Geografia pela Universidade Estadual de Santa Cruz, Especialização em Modelagem em Ciências da Terra e do Ambiente pela Universidade Estadual de Feira de Santana, Mestrado em Geografia pela Universidade Federal de Sergipe e Doutorado em Geociências e Meio Ambiente pela Universidade Estadual Paulista. Atualmente é docente da Universidade Estadual de Goiás. ORCID http://orcid. org/0000-0003-3814-3982 
Satélite Sino-Brasileiro de Recursos Terrestres na literatura científica global...

Patrick Thomaz de Aquino Martins . Pedro Paulino Borges

Pedro Paulino Borges - Tem graduação em Ciências Biológicas pela Universidade Estadual de Goiás- Unidade Universitária de Ciências Exatas e Tecnológicas. Mestre em Recursos Naturais do Cerrado pela Universidade Estadual de Goiás. Doutorado em andamento em Recursos Naturais do Cerrado. Possui experiência em coleta e análises de dados ecológicos. Atua em ecologia aquática, principalmente de lagoas de inundação e riachos. ORCID http:// orcid.org/0000-0003-2173-4276

Recebido para a publicação em 20 de março de 2020 Aceito para a publicação em 10 de maio de 2020 Publicado em 3 de junho de 2020 